\title{
The Mass Transfer Intensification of Combined Treatment Products
}

\author{
Oleg Skrygin ${ }^{1}$, Vladislav Smolentsev ${ }^{1 *}$, Anton Schednov ${ }^{1}$ \\ ${ }^{1}$ Voronezh State Technical University, 393026 Voronezh, 14 Moskovsky Prospekt, Russian \\ Federation
}

\begin{abstract}
The paper addresses the issues, related to mass transfer of treatment products when using combined forming methods with the application of an electric field. The impact of process components upon strength characteristics of parts has been shown. We have discovered the dependence of allowance removal rate upon the quality of preparation of the reference workpiece surface. The article describes the available industrial and newly developed methods of mass transfer intensification, using the original kinematics of movement, the improvement of the geometrical shape, the position of electrode-tools and the application of various options in the flow of liquid working media, including the cavitational mode, described in detail for the first time. We have disclosed the mechanism of the impulse effect upon treatment products, ejected from the inter-electrode gap by the liquid working medium. The issues have been discussed, concerning the hydrodynamic instability of liquid working media flow, impacting the intensity of mass transfer, with limitations, caused by the impact of side surfaces of holes and channels when processing with a profiled and a non-profiled wire electrode-tool. We have shown the impact of a concentrated ultrasonic beam upon mass transfer of treatment products, formed at a great distance from the electrode.
\end{abstract}

\section{Introduction}

The accumulation of treatment products in the inter-electrode gap limits the speed of material removal from a workpiece, which can quickly lead to complete cessation of allowance removal from it. This phenomenon is often observed with electrical methods of processing, especially, with combinations of thermal, chemical and mechanical impacts, used in electroerosive, electrochemical, dimensional and ultrasonic processing [1;2]. The literature $[3 ; 4 ; 5 ; 6]$ describes a variety of techniques, accelerating the mass transfer when using the processing methods under review, which, in most cases, intensifies the process, however, dramatically complicates its management and results in unreasonable cost increase of the operation. Therefore, there is an objective need for rational use of available types of mass transfer intensification of treatment products, and for the search of new ones, especially in manufacturing the parts of newly created equipment, extensively employing hard-to-process materials, for which the application of electrical processing methods is

${ }^{*}$ Corresponding author: vsmolen@inbox.ru 
most perspective.

\section{The features of the working medium flow through the inter- electrode gap}

The intensity of the transfer of treatment products depends on the geometry of the interelectrode gap profile. The fig. 1 shows the most commonly used schemes for transition of liquid working media through the treatment zone. In their planning, the main criterion of efficiency is the provision for the required mass transfer of treatment products.

The flow between:a)-flat surfaces;b)-curved sections;c)-the walls of the channel and the profile electrode with the rotation of the flow; d) the walls of the groove and non-profiled zlektrodom-wire with the output of the processing products in the groove obtained after the separation of the workpiece. 1.Electrode-tool.2.Billet.3.Direction of flow of the working medium.4.Processing products.

When the working medium flows along a flat surface (fig.1a), the resistance to liquid flow is minimal, which creates the conditions for emergence of a laminar stream with stable parameters and a uniform mass transfer of treatment products. However, the flat parts of workpieces processed represent only a small portion of the majority of modern products, and, in this case, the application of electrical processing methods is negligible.

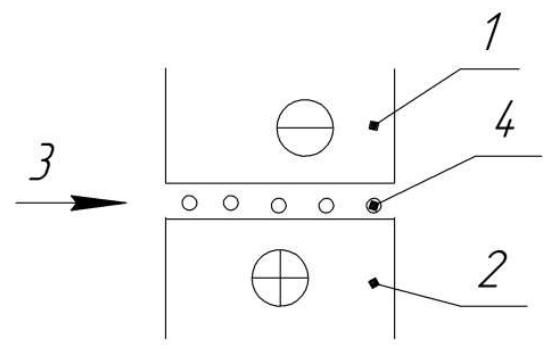

a

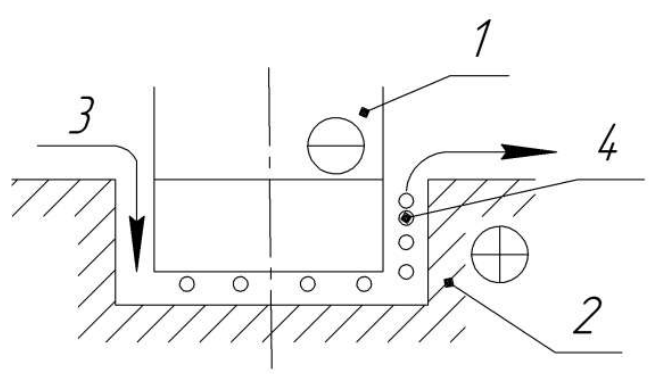

[

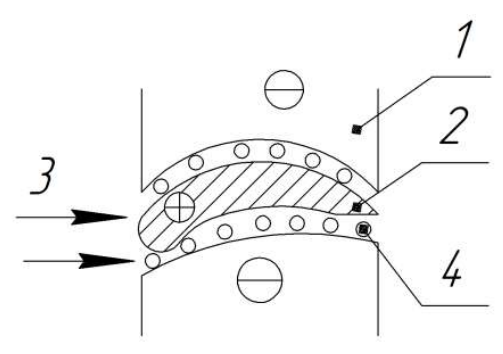

b

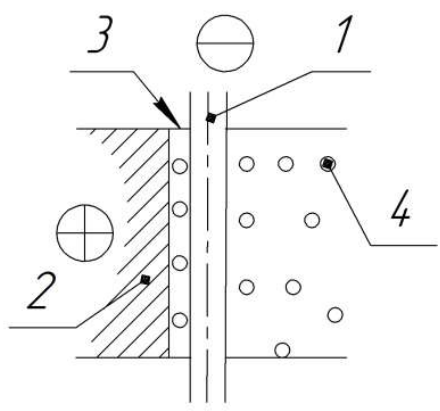

d

Fig. 1. Flow Diagrams of the liquid working medium through the interelectrode gap.

With sophistication in the shape of the surface processed, for example, during the manufacture of turbine and compressor blade airfoils in aviation, rocket and power engineering (fig.1b), the electrical processing methods are most applicable, especially in the case of aerodynamic geometry of the working path. Here, the required mass transfer of treatment products (4) from the inter-electrode gap (a tool electrode 1 - a workpiece 2 ) is 
obtained at low rates of the working medium flow (3, as shown in fig. 1b), and at low pressures at the liquid inlet into the gap. The main constraint on the application of electric processing methods is the track elongation until jetting, which causes the emergence of stagnant zones with reduced mass transfer, impairing the accuracy and quality of the surface layer of a part. In some cases, this causes the faults of expensive components. The fig. 2 shows the fragments of blades, manufactured from various materials with distorted profiles due to emergence of stagnant zones and consequent reduction in the mass transfer of treatment products.

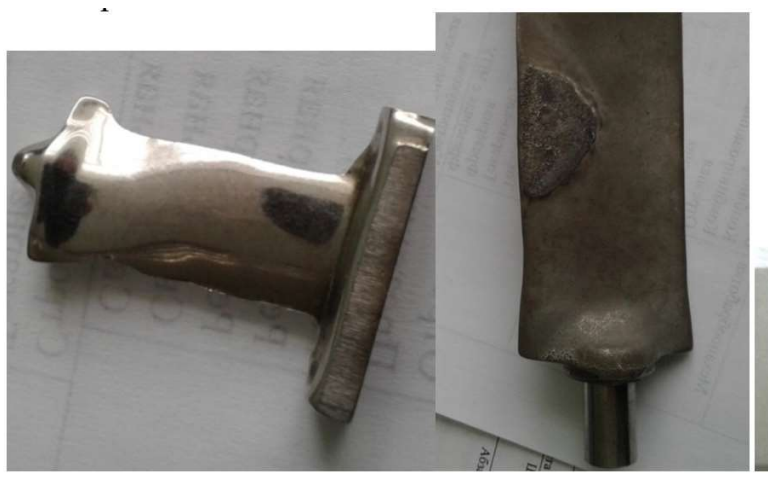

a) b)

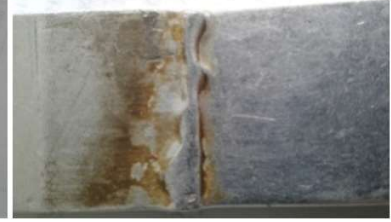

c)

Fig.2. Fragments of blades with profile violation due to the appearance of stagnant zones: a)-stainless steel alloy;b)-titanium alloy;c)- a sample of the pen blade aluminum alloy.

The irregularities, shown in fig. 2, significantly exceed the limits of variation in the thickness of a blade airfoil. The fig. 2 demonstrates both the protrusions in the lower part of an airfoil (this defect can be eliminated by local processing) and indents on the profile (dark areas), which can not be removed. The part was rejected as faulty. On the titanium part (fig. $2 \mathrm{~b}$ ), the protrusion exceeds $0.3 \mathrm{~mm}$ for the profile thickness tolerance of $0.1 \mathrm{~mm}$. The removal of the protrusion proved to be inexpedient due to formation of an indent of up to $0.5 \mathrm{~mm}$ around it (apparently, through accelerated ambient flow), which also resulted in the fault of the part.

The disturbance of the mass transfer is particularly characteristic in the section of an aluminium part, (as shown in fig.2), in the place of its fixation in the device, where both serious faults and micro-surface defects in the form of profile waviness are clearly seen.

In the process of testing the manufacturability, the defects, similar to those, described in fig. 2 for blades with an airfoil thickness of up to $50 \mathrm{~mm}$, were completely eliminated by improving the geometry of inlet sections of channels and regulating the pressure of the working medium. However, the problem of mass transfer exists only in case when a part has an increased length of the processing zone and complex transitional areas, impeding the mass transfer. An example of such a flow of working liquid is shown in figure 1, where a blind or a through deep hole of any cross-section is made by a solid profiled electrode 1 in the workpiece 2 . In any case, the direction 3 of the flow drastically changes in the bottom part, where stagnant zones and jet streams occur, impairing the workpiece quality and reducing the performance of piercing by an order of magnitude and more. It is possible to partially restore the mass transfer in such an operation through increasing the pressure of the input working medium by up to 10 times, however, the results achieved ensure the hole piercing in steels to depths of less than 10-15 times the hole diameter.

It is particularly difficult to organize the mass transfer of multi-electrode hole treatment products, for example, in filters, when it is required to pierce up to 500 deep holes at a time with the minimum bridge. In this case, the available methods of mass transfer 
intensification do not provide the speed of piercing, attained for a single profiled electrode. Some of the new technological solutions for mass transfer intensification are presented below in this article.

The constraints on mass transfer when using the processes of material separation by machines with a non-profiled wire electrode (fig. $1 \mathrm{~g}$ ), most commonly used in industry, limit the ultimate thickness of workpieces being cut. The jet injection of liquid into the processing zone helped to increase the depth of processing, however, it is necessary to create new methods $[7 ; 8 ; 9]$, providing mass transfer with increased values of workpiece thickness.

\section{The methods of mass transfer intensification}

In the literature [9], different methods of removing the treatment products from the process zone are described. The efficiency of each method is evaluated by the efficiency of mass transfer, contingent on many factors. The schemes of implementing the methods are presented in fig. 3 .

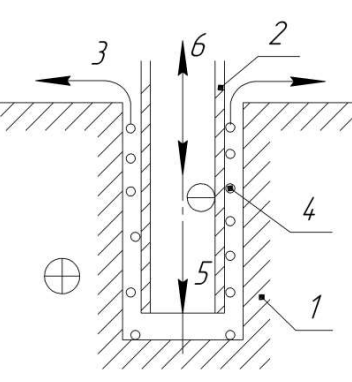

a

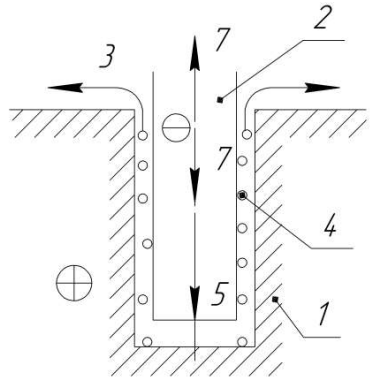

b

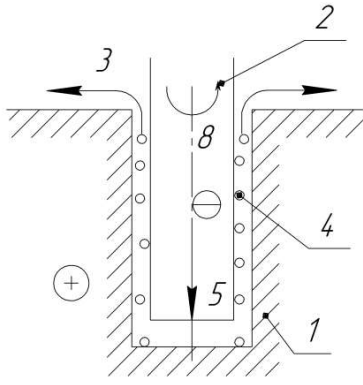

ᄃ

Fig.3. The methods of mass transfer intensification during hole piercing a) - by creating a pumping effect; b) - by periodic withdrawal of a profiled tool electrode from a hole; c) - by rotation of the profiled tool-electrode: 1 - a workpiece; 2 - a tool electrode; 3 - liquid working medium; 4 treatment products; 5 - the feed direction of the tool electrode; 6 - the directions of vibrational movements of the tool electrode; 7 - the directions of the input and output from the pierced part of holes of a profiled tool electrode; 8 - the direction of the rotary motion of the electrode tool.

The fig. 3 shows the method of mass transfer acceleration due to the pumping effect from the longitudinal vibration of the tool electrode (the amplitude is $0,05-0,2 \mathrm{~mm}$, the frequency is $100-120 \mathrm{~Hz}$ ), extensively used in industry with electrical processing methods [1;3]. At a sufficient speed of the longitudinal tool electrode movement, the pumping effect and the displacement of treatment products (4) in the gap create the cavitational flow of the working medium, drastically increasing the mass transfer, the performance of processing and the surface layer quality. According to [3], the marginal ratio of the hole depth to diameter during piercing can not exceed $10-15$, which does not always satisfy the industrial needs.

Deeper holes are obtained by periodical withdrawal (7) of the tool electrode 2 from the hole (fig. 3b) (in some cases, using vibration, according to the scheme, shown in fig.3a), where the pumping effect is enhanced and no time is required for cleansing the pierced part of the hole from treatment products (4). This makes it possible to increase the ultimate depth of piercing up to 20-25 with significant (up to 1.5-2 times) reduction in the process performance.

As shown in fig. 3, the mass transfer acceleration is achieved by generation of a 
helical flow of the working medium during the tool electrode (2) rotation (8) (fig.3c) (sometimes, in combination with methods a, b). Unfortunately, rotation is not applicable during multi-electrode processing of non-circular holes, especially those of small crosssections.

In [64849], it is shown that, in processing long workpieces with anodic allowance dissolution, the accumulation of gaseous treatment products can block the inter-electrode gap, with all the methods, presented in fig.3, becoming ineffective. In this case, not only the performance, but also the surface layer quality of the part and the profile accuracy are getting worse. To eliminate the constraints on mass transfer intensity, it is proposed in [ $8 ; 9]$ to apply the cyclical allowance removal. The fig. 4 shows the sequence diagram for tuning the mechanism of system control with respect to titanium blades with airfoil thickness of $180 \mathrm{~mm}$.

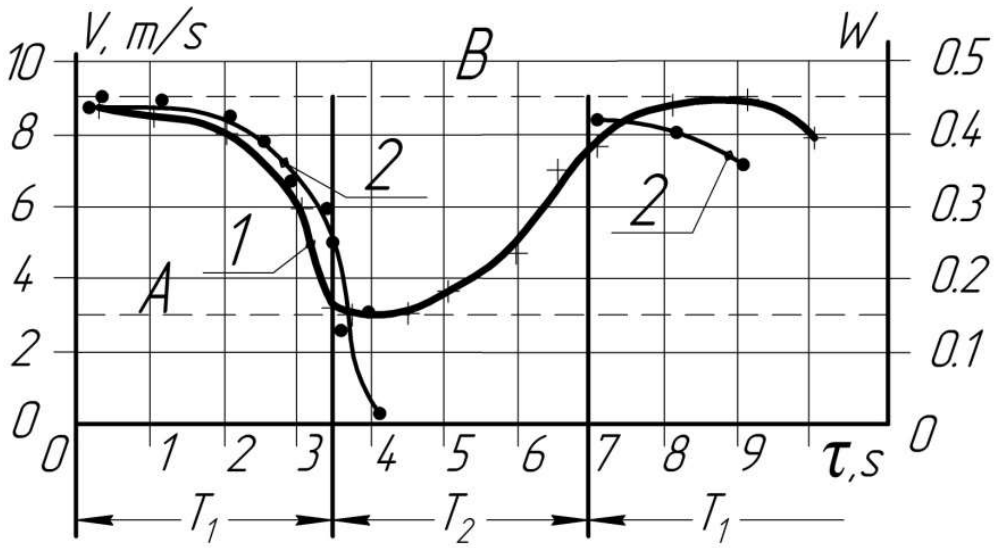

Fig.4. The change in the average speed of working medium flow (V) and allowance removal $(\mathrm{W} \tau)$ with cyclical supply of process current over time $(\tau)$ T1- the period of anodic dissolution of allowance by process current; $\mathrm{T} 2$ - the period of treatment product removal from the inter-electrode gap with no anodic allowance dissolution. 1. - the average speed of working medium flow over a period, $\mathrm{m} / \mathrm{sec} ; 2$ - the average speed of allowance removal over a period $\mathrm{T} 1+\mathrm{T} 2, \mathrm{~mm} / \mathrm{min}$. $\mathrm{A} ; \mathrm{B}-$ the limits of parameter variation

The method of cyclical processing, shown in fig.4, makes it possible to stabilize the mass transfer and, in many respects, eliminates the constraints on processing of workpieces with very long paths of working medium flow, typical for large-size parts.

\section{The perspective methods of mass transfer intensification when using the electrical processing methods}

The works [7;10] describe new (as a rule, at the level of inventions) methods and devices for mass transfer intensification for various types of electrical processing methods, including those, which involve the impulse effect of an ultrasonic field ..

The fig. 5 presents the scheme of combined formation of deep channels in a dielectric workpiece. 


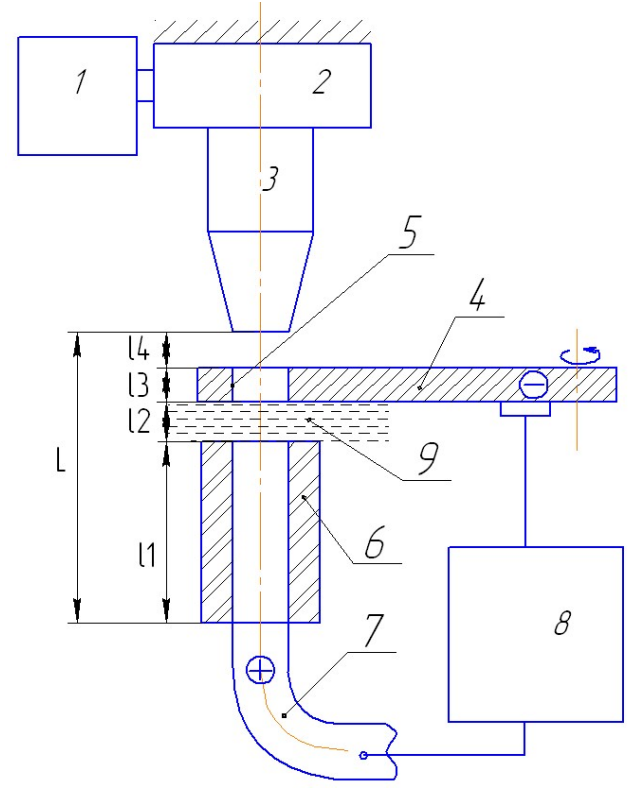

Fig.5. The scheme of combined processing of channels in a mineral-ceramic workpiece: 1- an ultrasonic generator; 2 - a converter; 3 - a radiator; 4 - a cathode; 5 - a hole for passing of an ultrasonic beam; 6 - a mineral-ceramic plug; 7- a metal insert; 8 - a generator of current for electrochemical processing; 9 - electrolyte; 10-14 - the parts of the system, specifying the interelectrode gap L- the greatest length of the inter-electrode gap

The fig. 5 shows the scheme of the new method [7] of combined channel processing in a nozzle by removing the metal insert 7 , embedded in the mineral-ceramic plug 6 before pressing, and having a profile, specified by the customer and the designer of the product. The shape of cross-sections in channels and the directions of their axes can vary, and the axes of holes can be straight or curved.

The method is effective in producing precise channels in locations of parts, inaccessible for the tool feed. The minimal diameter of holes is less than $0.1 \mathrm{~mm}$, and the depth is up to 100 times the diameter. The development of this method due to mass transfer intensification is presented in [3].

\section{Conclusion}

The materials, presented in the article, make it possible to outline the ways of improving and expanding the field of using electrical processing methods, based on well-known and newly developed methods of mass transfer intensification of process products. This ensures the stability of obtaining the required performance characteristics of parts and offers the opportunities of extensive use of combined processing methods in high-tech engineering industries.

\section{References}

1. A.B. Bondar, V.P. Smolentsev, A.I Chasovskih Addives, Frame and control of an engineering control in engineering (Oxford, 2001)

2. A.B Bondar, G.A. Sukhochev V.P. Smolentsev, State of a surface layer after processing with combined effect (EM - 06, Polska, Bydgoszcz, 2006) 
3. V.P. Smolentsev, S.V. Safonov, E.V. Smolentsev, IOP Conf. Ser.: Mater. Sci. Eng., 124, 01214 (2016)

4. S.V. Safonov, V.P. Smolentsev, Nauka i studia. V international conference «Science and education», 57 (2014)

5. M.A. Tamarkin, E.E. Tichenko, V.G. Lebedenko, Russian Engineering Research, 10, 2, 144 (2010)

6. V.A Lebedev., V.V. Ivanov, V.P. Fedorov, IOP Conf. Ser.: Mater. Sci. Eng. 124, 012160 (2016)

7. V.P. Smolentsev, E.V. Smolentsev, B. I. Omigov, Yu. V. Sharov, Patent 2680327 (The Russian Federation, 2019)

8. A. P. Babichev, B.S., Glazman, D.N. Kravchenko, V.V. Ivanov, 7lh International Conference on Deburring and Surface Finishing (University of California, Berkley, 2004)

9. V.V. Ivanov, V.A. Lebedev., I. A .Pinahin, Jornal of Friction and Wear, 35:4, 339 (2014)

10. V.P. Smolentsev, E. A. Saltanaeva, E.V. Smolentsev, I. I. Koptev, N. V. Pishkova , patent 2537410 (The Russian Federation, 2015) 